\title{
NOTES
}

\section{Phylogeny of Methanopyrus kandleri Based on Methyl Coenzyme M Reductase Operons}

\author{
JÖRK NÖLLING, ${ }^{1}$ AMY ELFNER, ${ }^{1}$ JOHN R. PALMER, ${ }^{1}$ VANESSA J. STEIGERWALD, ${ }^{1}$ \\ TODD D. PIHL, ${ }^{1}$ JAMES A. LAKE, ${ }^{2}$ AND JOHN N. REEVE ${ }^{1 *}$ \\ Department of Microbiology, The Ohio State University, Columbus, Ohio $43210^{1}$ and Molecular \\ Biology Institute, University of California, Los Angeles, California $90024^{2}$
}

\begin{abstract}
The mcrBDCGA operon that encodes methyl coenzyme $M$ reductase (MR) in the hyperthermophile Methanopyrus kandleri was cloned and sequenced. The results of a phylogenetic analysis of the nine MR sequences now available support the position that $M$. kandleri is a separate methanogen lineage. As in other methanogens, the $M$. kandleri mor operon is located immediately upstream of the $m t r E$ gene, the promoter-proximal gene in an operon that encodes the $N^{5}$-methyltetrahydromethanopterin:coenzyme $M$ methyltransferase that catalyzes the step preceding the MR-catalyzed reaction in methanogenesis. In contrast to other methanogens and hyperthermophilic members of the Archaea, CG dinucleotides and CG-containing codons occur frequently in M. kandleri DNA. The MR subunit-encoding genes are preceded by sequences consistent with ribosome binding sites, indicating that mRNA-rRNA base pairing can still direct translation initiation in cells growing at temperatures above $100^{\circ} \mathrm{C}$.
\end{abstract}

Methanogens provide a model system for microbial phylogenetics. On the one hand, they are extremely diverse, with genomes whose $\mathrm{G}+\mathrm{C}$ contents range from 23 to $61 \mathrm{~mol} \%$, with bacillary, coccal, and spiral morphologies and a variety of cell envelope structures, and with habitats as different as the rumen and submarine volcanic vents $(1,2,8,9,14,16)$. On the other hand, they are unified (and named) by their ability and need to synthesize methane. Facultative methanogens are unknown, and the final reaction in methanogenesis, the reduction of a methyl group bound to coenzyme $M$, releasing methane, is common to all methanogens (8). Methyl coenzyme $M$ reductase (MR), the enzyme that catalyzes this reaction, has been purified from many methanogens and consistently has an $\alpha_{2} \beta_{2} \gamma_{2}$ polypeptide subunit composition (11). The genes that encode these subunits, $m c r A, m c r B$, and $m c r G$, respectively, have been cloned and sequenced from several different methanogens and have always been found to be arranged $m c r B D$ $C G A$, within a single transcriptional unit, designated an MR operon $(18,25,33)$. The functions of the $\operatorname{mcr} C$ and $m c r D$ gene products remain unknown (31). Some methanogens contain a second MR isoenzyme (MRII) (27), and two MRII-encoding operons ( $m r t$ [MR two]) have also been cloned and sequenced $(21,24)$. These $m r t B D G A$ operons differ from the $m c r$ operons in lacking a " $C$ " gene but otherwise are very similar and clearly evolved from the same common ancestor as the $\mathrm{mcr}$ operons. In 1988 Weil et al. (33) proposed that because the basic structure and function of MR are conserved in all methanogens, changes in the sequences of the MR subunits should provide valid estimates of the evolutionary divergence of methanogens, and recently Springer et al. (30) provided experimental support for this concept. A phylogenetic tree for the Methanosarcinaceae was constructed on the basis of the sequences of the same 490-bp region amplified from 25 different $m c r A$ genes, and this tree was fully consistent with the phylogeny of these

* Corresponding author. Phone: (614) 292-2301. Fax: (614) 2928120. Electronic mail address: jreeve@magnus.acs.ohio-state.edu. methanogens established previously on the basis of their $16 \mathrm{~S}$ rRNA sequences.

The position of Methanopyrus kandleri is particularly interesting for methanogen phylogenetics (26). Methanopyrus kandleri has the most divergent $16 \mathrm{~S}$ rRNA sequence, genomic DNA with an unusually high $\mathrm{G}+\mathrm{C}$ content $(60 \mathrm{~mol} \%)$, and the highest known optimum growth temperature $\left(98^{\circ} \mathrm{C}\right)$ and grows at temperatures up to $110^{\circ} \mathrm{C}(3,13,19)$. These features suggest that the lineage of this organism diverged very early from the other methanogen lineages, but Methanopyrus kandleri is nevertheless a methanogen. It should therefore have a phylogenetically informative MR operon, and in this paper we describe the cloning, sequence, and results of a phylogenetic analysis of the Methanopyrus kandleri mcr operon. The data obtained in this study also provide evidence that unusual codon usage occurs and that mRNA-tRNA base pairing is used in Methanopyrus kandleri cells to direct translation initiation at temperatures up to $110^{\circ} \mathrm{C}$.

On the basis of the highly conserved sequences (33) used as hybridization probes for $m c r B$ and $m r t B$ transcripts (24), we designed and used GC-rich primers to PCR amplify a 133-bp region from within the Methanopyrus kandleri $m c r B$ and/or $m r t B$ genes. Molecules from the pool of amplified DNA were cloned into pUC19, and several molecules were sequenced (28), which confirmed that they were identical and were from a $m c r B$ gene. Southern blot hybridizations (28) with Methanopyrus kandleri genomic DNA revealed that the PCR-amplified region was located within an $\sim 15$-kbp EcoRI fragment, and a $\lambda$-gt11 recombinant phage that contained this fragment intact was identified, by plaque hybridization (28), in a Methanopyrus kandleri genomic library. This EcoRI fragment was subcloned, and from the resulting subclone and additional subclones we sequenced the MR-encoding genes and flanking regions. The sequence obtained (GenBank accession number U57340) contained open reading frames consistent with the presence of a standard $m c r B D C G A$ operon located upstream from an $m t r E$ gene (Fig. 1A). In other methanogens, the $m t r E$ 
A.

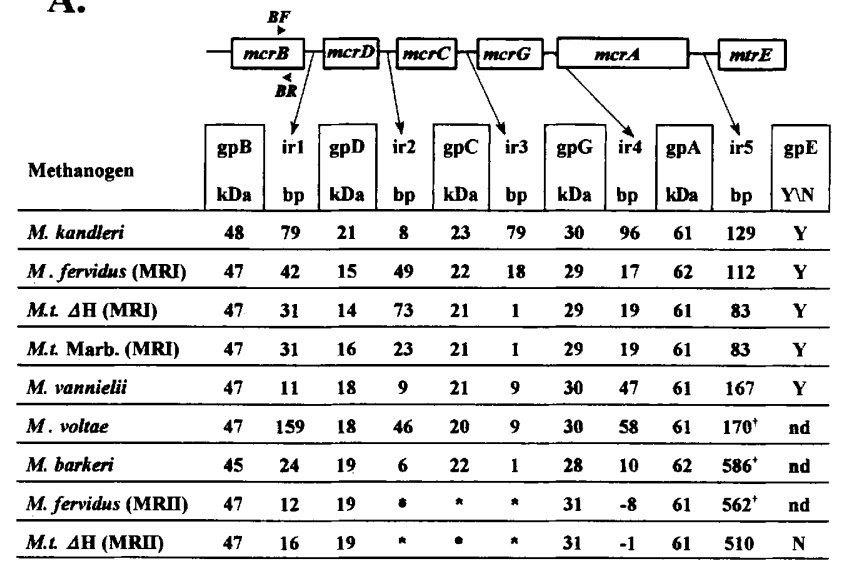

B.

\section{5'-Upstream regions:}

\begin{abstract}
5. AGTATTTAAAAGTGAGATCAAGTGCTCGATAGGGGTTCAAACAATGG-mer 5 ' GATGACGCATTGGTAAAGATACCCTAACACGGGGTGCCACGCATGG-mth 5 ' GATATTAAAGGGCCAGGTCGGGTGGCTCCTGCGGGGTGTTCCGGGTGG- $f t r$ 5 ' GTTCACTTTCGTAGCTCTCAACTCGAGTTTAGGGGGTACGCCCTTGG-mtrE 5 ' ACCCTTTAAAGCTGCAGCCCTGCGAAGTTAGGGGGGCCCGCTTATGG-mCr $B$ 5 ' AGGGGGCGCGACCTTGCCGGAGGAGAAACTCGAGCGGCCCCGCATGT-mCrD 5 'GGGTCTCCGTCCTCAAGAAGAAGGGGGAGGACTAACCTCGCGAGTGG-mcrC 5 ' CCСTCACACTTCAGGGGCTGACCTTATGGGTCTGAAGGAGGAGATGG-mCrG

5 ' TATAACGCTCTCGACCTATCGCCCCATGGGGGAGAGGATCACGATGT-mCrA
\end{abstract}

FIG. 1. Structure and comparison of the Methanopynus kandleri mcr operon and gene products. (A) A 5,914-bp sequence (GenBank accession number U57340) was determined, and this sequence contained the entire Methanopyrus kandleri $m c r B D C G A$ operon and 231 bp of the $m$ trE gene, organized as shown at the top. The locations of the two sequences used as PCR primers ( $m c r B$ forward primer $\mathrm{BF}$ and $m c r B$ reverse primer BR) to amplify the DNA fragment used as the hybridization probe are indicated. Also shown are the calculated molecular masses (in kilodaltons) of the sequenced $m c r$ (MRI) and $m r t$ (MRII) gene products (gp) and the lengths (in base pairs) of the intergenic regions (ir) (5, 18, $21,24,33$ ). The absence of an $m r t C$ gene is indicated by asterisks. If the first open reading frame downstream from the $\alpha$-subunit-encoding gene ( $m c r A$ or $m r t A$ ) has been shown to be $m t r E$, this is indicated ( $\mathrm{Y}$, yes; $\mathrm{N}$, no) under $\mathrm{gpE}$. nd indicates that an $m t r E$ gene has not been detected and no open reading frames have been identified within the ir $5^{\dagger}$ designated regions. (B) Intergenic sequences immediately upstream of $\mathrm{mcr}$ and the three other Methanopyrus kandleri methane genes sequenced $(23,29,34)$ aligned, at the arrow, by their translationinitiating codons. Start codons, putative ribosome binding sites, and archaeal TATA-box promoter elements are identified by the boldface type, and the TAA termination codon of the $m c r D$ gene is underlined. $M$. kandleri, Methanopyrus kandleri; $M$. fervidus, Methanothermus fervidus; M. $t . \Delta \mathrm{H}$, Methanobacterium thermoautotrophicum $\Delta \mathrm{H} ;$ M. t. Marb., Methanobacterium thermoautotrophicum Marburg; $M$. vannielii, Methanococcus vannielii; $M$. voltae, Methanococcus voltaei; M. barkeri, Methanosarcina barkeri.

gene is the promoter-proximal gene in an $m$ tr operon that encodes $N^{5}$-methyltetrahydromethanopterin:coenzyme $\mathrm{M}$ methyltransferase $(10)$. This enzyme catalyzes the penultimate step in methanogenesis, and the same adjacent positioning of the $m c r$ and $m t r$ operons has also been found in the genomes of Methanococcus vannielii, Methanobacterium thermoautotrophicum, and Methanothermus fervidus (22, 24, 33). As growth phase-dependent read-through transcription of the $m$ tr operon from the mor promoter has been documented in Methanobacterium thermoautotrophicum, this neighborhood arrangement of methane gene operons (24) appears to have functional significance. Some Southern blots adumbrated the existence of a second MR-encoding region in the Methanopyrus kandleri genome, but despite a substantial effort, we were unable to clone directly or to PCR amplify an mrt operon from Methanopyrus kandleri.

The sizes of the MR $\alpha, \beta$, and $\gamma$ subunits are very similar in all methanogens (11), including Methanopyrus kandleri (Fig. 1A), whereas the intergenic regions within and downstream of the different MR operons vary in length and have divergent sequences (Fig. 1A) (18, 33). Methanogens synthesize large amounts of MR $(8,11,27)$, and sequences consistent with the presence of very strong ribosome binding sites have been identified upstream of the previously sequenced $m c r A, \operatorname{mcr} B$, and $m c r G$ genes $(2,25)$. In Fig. $1 \mathrm{~B}$, all of the sequences so far determined upstream of Methanopyrus kandleri methane genes are aligned, and the data demonstrate that in every case there is at least one $5^{\prime}$-GGGG and/or 5'-GGAGG sequence, appropriately positioned for a ribosome binding site, that could base pair with the sequence HO-UCCUCCACU-5' at the $3^{\prime}$ terminus of the Methanopyrus kandleri 16S rRNA (3). The consistency and locations of these sequences argue that they do function as ribosome binding sites $(2,25)$ which, in turn, argues that rRNA-mRNA base pairing must be possible inside Methanopyrus kandleri cells growing at $110^{\circ} \mathrm{C}$. These sequences are, however, somewhat shorter and offer fewer opportunities for consecutive base pairing with the $16 \mathrm{~S}$ rRNA than do their counterparts in other methanogens, suggesting that rRNAmRNA interactions in Methanopyrus kandleri must also be facilitated and stabilized by mechanisms in addition to spontaneous Watson-Crick base pairing.

Codon usage in the MR subunit-encoding genes is also consistent with the high levels of expression of these genes (18, 33). Each of the synonymous codon pairs $A A C / U, G A C / U$, AUC/U, UUC/U, and UAC/U is translated by one tRNA, and all of these $t R N A$ s have a $G$ in the first anticodon position. The previously sequenced $m c r A / m r t A, m c r B / m r t B$, and $m c r G / m r t G$ genes preferentially use the codons with the $C$ residue in the wobble position, which results in $\mathrm{G} \cdot \mathrm{C}$ rather than $\mathrm{G} \cdot \mathrm{U}$ codon anticodon base pairs. This is expected to maximize the rate of translation of the transcripts (32). The $m c r C$ and $m c r D$ gene products, in contrast, are not synthesized in large amounts (31), and the $m c r C$ and $m c r D$ genes do not exhibit this preferential use of the C-containing codons from these synonymous pairs $(18,33)$. The overall $\mathrm{G}+\mathrm{C}$ content of Methanopyrus kandleri genomic DNA is $60 \mathrm{~mol} \%$; therefore, codons with a $\mathrm{C}$ in the wobble position would be expected to occur frequently, and this is the case. The Methanopyrus kandleri mcrC and $m c r D$ genes contain 44 members of the synonymous codon pairs, and 40 of these have a $\mathrm{C}$ in the wobble position. There is therefore a 10:1 ratio in favor of the C-containing codons over the U-containing codons in these genes. In the MR subunit-encoding genes there are $228 \mathrm{C}$-containing codons and only $7 \mathrm{U}$-containing codons from these pairs; i.e., the ratio in these genes is $32: 1$ in favor of the C-containing codons. Thus, the increased use of the C-containing codons in the $m c r A$, $m c r B$, and $m c r G$ genes compared with the $m c r C$ and $m c r D$ genes in Methanopyrus kandleri is 3.2-fold, which is consistent with the range of 2.2- to 5.7-fold increased usage of C-containing codons found in other methanogens (33).

The dinucleotide 5'CG occurs very frequently in Methanopyrus kandleri DNA. This codon is the most abundant dinucleotide in the $m c r$ operon, accounting for $\sim 13 \%$ of all dinucleotides and contributing to the presence of 238 CG-containing codons. In contrast, the CG dinucleotide and CG-containing codons occur only rarely in other methanogens and nonmethanogenic hyperthermophiles (6). There are only 26, 31, 46, 26, 20 , and 12 CG-containing codons in the $\mathrm{mer}$ operons of Methanobacterium thermoautotrophicum $\Delta \mathrm{H}$ and Marburg, Methanosarcina barkeri, Methanothermus fervidus, Methanococcus 


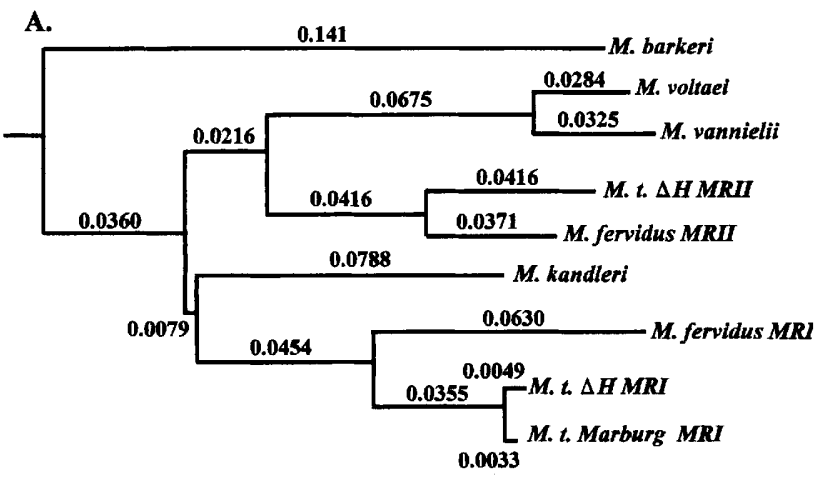

B.

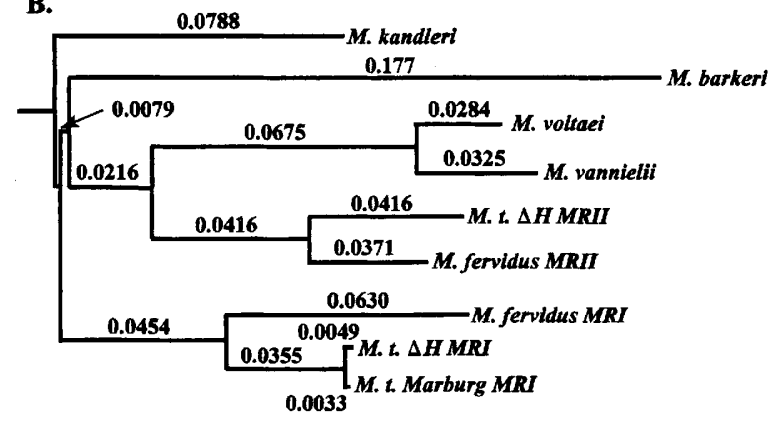

FIG. 2. Phylogenetic trees constructed by paralinear distance analysis of the sequences of the $\alpha, \beta$, and $\gamma$ subunits of MRI and MRII in Methanosarcina barkeri (M. barkeri), Methanococcus voltaei (M. voltaei), Methanococcus vannielii (M. vannielii), Methanobacterium thermoautotrophicum (M. t. $\Delta \mathrm{H})$, Methanobacterium thermoautotrophicum Marburg (M. t. Marburg), Methanothermus fervidus (M. fervidus), and Methanopyrus kandleri (M. kandleri). The branch lengths, in number of nucleotide substitutions per position (20), are indicated. (A) Tree rooted by assuming that the rates of nucleotide substitution were the same in the $m c r$ and $m r t$ genes in all of the methanogen lineages. (B) Tree rooted on the basis of the conclusion from 16S rRNA studies that Methanopyrus kandleri is the most divergent methanogen $(3,13,19)$. The most probable unrooted trees generated from the sequences by maximum-parsimony ( $94 \%$ probability), Jukes-Cantor ( $96.5 \%$ probability), and Kimura $(96 \%$ probability) distance analyses $(15,17)$ had the same branching topologies.

vannielii, and Methanococcus voltaei, respectively. CG-containing sequences are frequently targets for DNA cytosine methylations, and methyl-cytosines have a high propensity for spontaneous deamination, especially at high temperatures (7), so that nonessential CG-containing sequences in cells containing DNA cytosine-methylating enzymes should eventually become TG-containing sequences (4). It appears, therefore, that Methanopyrus kandleri cells must lack such DNA cytosine-methylating enzymes or contain mechanisms that prevent or correct methyl-cytosine deaminations.

To construct phylogenetic trees based on the MR subunit data, the amino acid sequences of all of the available $\alpha, \beta$, and $\gamma$ subunits were aligned, and after regions of uncertain alignment were discarded, the remaining sequences were backtranslated into nucleotide sequences. Third codon positions were then eliminated, and only uniquely defined first- and second-position nucleotides were used to calculate the paralinear distances (20) that were used to generate the trees shown in Fig. 2. Unrooted trees with the same branching topology were also obtained when the alignments were subjected to maximum-parsimony, Jukes-Cantor, and Kimura distance analyses $(15,17)$. Because MR occurs only in methanogens, it was impossible to root these trees with an outgroup, and therefore we constructed two trees, one rooted by assuming that nucleotide substitution rates in the $m c r$-mrt genes were the same in all lineages (Fig. 2A) and one rooted in the Methanopyrus lineage, as indicated by the 16S rRNA studies (Fig. 2B) (3). Both trees contain Methanopyrus kandleri in a separate and very divergent lineage, but if the tree is rooted, as in Fig. 2A in the Methanosarcina barkeri lineage, then the Methanopyrales and Methanobacteriales are related as neighbors, which is consistent with the similar cell wall structures of these groups (16).

Surprisingly, the $m r t$ genes (MRII) in the members of the Methanobacteriales are more similar to the methanococcal $\mathrm{mcr}$ genes (MRI) than they are to their own $m c r$ genes $(21,24)$. The methanococcal $\mathrm{mcr}$ operons could therefore be $m r t$ operons that still contain an $m r t C$ gene; however, this is difficult to reconcile with the neighborhood organization. Immediately downstream from the Methanococcus vannielii mcr operon is an $m t r$ operon (22), as is also the case in the genomes of Methanopyrus kandleri, Methanobacterium thermoautotrophicum and Methanothermus fervidus (Fig. 1A). Alternatively, the $m r t$ operons could have evolved after lateral gene transfer of an $m c r$ operon from a member of the Methanococcales into the ancestor of the extant Methanobacteriales (24), or the operon duplication that gave rise to the $m c r$ and $m r t$ operons could have occurred before the divergence of the Methanococcales and Methanobacteriales (21). If the latter explanation is correct, then the evidence against the presence a second MR-encoding region in Methanococcus vannielii (12) suggests that members of the Methanococcales subsequently lost one MR-encoding region. As a phylogenetic study of 25 amplified $m c r A$ sequences (30) also failed to find any evidence for a second MR-encoding region in the members of the Methanosarcinaceae, this lineage may also have lost the second MR-encoding region. The Methanosarcinaceae could, alternatively, have diverged from all of the other methanogen lineages before the operon duplication occurred, which would be consistent with the phylogenetic tree shown in Fig. 2A. If there is also an $m r t$ operon in Methanopyrus kandleri, this would provide additional parsimonious support for the phylogeny shown in Fig. $2 \mathrm{~A}$, but it would still not eliminate the possibility that the original methanogen contained both an mcr operon and an $m r t$ operon.

This research was supported by National Science Foundation grant DMB-9002729 and by Department of Energy grant DE-FG0287ER13731.

We thank K. O. Stetter for the gift of Methanopyrus kandleri cells.

\section{REFERENCES}

1. Balch, W. E., G. F. Fox, L. J. Magrum, C. R. Woese, and R. S. B. Wolfe. 1979. Methanogens: re-evaluation of a unique biological group. Microbiol. Rev. 43:260-296.

2. Brown, J. W., C. J. Daniels, and J. N. Reeve. 1989. Gene structure, organization and expression in archaebacteria. Crit. Rev. Microbiol. 16:287-338.

3. Burggraf, S., K. O. Stetter, P. Rouviere, and C. R. Woese. 1991. Methanopyrus kandleri: an archaeal methanogen unrelated to all other known methanogens. Syst. Appl. Microbiol. 14:346-351.

4. Coulondre, C., J. H. Miller, P. J. Farabaugh, and W. Gilbert. 1978. Molecular basis of base substitution hotspots in Escherichia coli. Nature (London) 274:775-780.

5. Cram, D. S., B. A. Sherf, R. T. Libby, R. J. Mattaliano, K. L. Ramachandran, and J. N. Reeve. 1987. Structure and expression of the genes, $\operatorname{mcr} B D C G A$, which encode the subunits of component $C$ of methyl coenzyme $M$ reductase in Methanococcus vannielii. Proc. Natl. Acad. Sci. USA 84:3992-3996.

6. DiRuggiero, J., K. M. Borges, and F. T. Robb. 1995. Codon usage tables for thermophilic archaea, p. 191-194. In F. T. Robb, A. R. Place, K. R. Sowers, H. J. Schreier, S. DasSarma, and E. M. Fleishmann (ed) Archaea: a laboratory manual. Cold Spring Harbor Press, Cold Spring Harbor, N.Y.

7. Ehrlich, M., M. A. Gama-Sosa, L. H. Carriera, L. G. Ljundahl, K. C. Kuo, and C. W. Gehrke. 1985. DNA methylation in thermophylic bacteria: N ${ }^{4}$ methylcytosine, 5-methylcytosine, and $\mathrm{N}^{6}$-methyladenine. Nucleic Acids Res. 13:1399-1412.

8. Ferry, J. G. 1993. Methanogenesis. Ecology, physiology, biochemistry and genetics. Chapman \& Hall, New York. 
9. Garcia, J. L. 1990. Taxonomy and ecology of methanogens. FEMS Microbiol. Rev. 87:297-308.

10. Harms, U., D. S. Weiss, P. Gärtner, D. Linder, and R. K. Thauer. 1995. The energy conserving $N^{5}$-methyltetrahydromethanopterin:coenzyme $\mathrm{M}$ methyl transferase complex from Methanobacterium thermoautotrophicum is composed of eight different subunits. Eur. J. Biochem. 228:640-648.

11. Hartzell, P. L., and R. S. Wolfe. 1986. Comparative studies of component C from the methyl reductase system of different methanogens. Syst. Appl. Microbiol. 7:568-577.

12. Hennigan, A. N., and J. N. Reeve. 1994. mRNAs in the methanogenic archaeon Methanococcus vannielii: numbers, half-lives and processing. Mol. Microbiol. 11:655-670.

13. Huber, R., M. Kurr, H. W. Jannasch, and K. O. Stetter. 1989. A novel group of abyssal methanogenic archaebacteria (Methanopyrus) growing at $110^{\circ} \mathrm{C}$. Nature (London) 342:833-834.

14. Jones, W. J., D. P. Nagle, Jr., and W. B. Whitman. 1987. Methanogens and the diversity of archaebacteria. Microbiol. Rev. 51:135-177.

15. Jukes, T. H., and C. R. Cantor. 1969. Evolution of protein molecules, p. 21-132. In H. N. Munro (ed.), Mammalian protein metabolism, vol. III. Academic Press, New York.

16. Kandler, O., and K. König. 1993. Cell envelopes of archaea: structure and chemistry, p. 223-259. In M. Kates, D. J. Kushner, and A. T. Matheson (ed.), The biochemistry of Archaea (Archaebacteria). Elsevier Science Publishers B.V., Amsterdam.

17. Kimura, M. 1983. The neutral theory. Cambridge University Press, London.

18. Klein, A., R. Allmansberger, M. Bokranz, S. Knaub, B. Müller, and E. Muth 1988. Comparative analysis of genes encoding methyl coenzyme $\mathrm{M}$ reductase in methanogenic bacteria. Mol. Gen. Genet. 213:409-420.

19. Knurr, M., R. Huber, H. König, H. W. Jannasch, H. Fricke, A. Trincone, J. K. Kristjansson, and K. O. Stetter. 1991. Methanopyrus kandleri, gen. and sp. nov. represents a novel group of hyperthermophilic methanogens, growing at $110^{\circ} \mathrm{C}$. Arch. Microbiol. 156:239-247.

20. Lake, J. A. 1994. Reconstructing evolutionary trees from DNA and protein sequences: paralinear distances. Proc. Natl. Acad. Sci. USA 91:1455-1459.

21. Lehmacher, A., and H.-P. Klenk. 1994. Characterization and phylogeny of $m c r I I$, a gene cluster encoding an isoenzyme of methyl coenzyme $\mathbf{M}$ reductase from hyperthermophilic Methanothermus fervidus. Mol. Gen. Genet. 243:198-206.

22. Nölling, J. 1996. Unpublished data

23. Nölling, J., T. D. Pihl, and J. N. Reeve. 1995. Cloning, sequencing, and growth phase-dependent transcription of the coenzyme $\mathrm{F}_{420}$-dependent $N^{5}$, $N^{10}$-methylenehydromethanopterin reductase-encoding genes from Meth- anobacterium thermoautotrophicum $\Delta \mathrm{H}$ and Methanopyrus kandleri. J. Bacteriol. 177:7238-7244.

24. Pihl, T. D., S. Sharma, and J. N. Reeve. 1994. Growth phase-dependent transcription of the genes that encode the two methyl coenzyme $M$ reductase isoenzymes and $N^{5}$-methyltetrahydromethanopterin:coenzyme $\mathrm{M}$ methyl transferase in Methanobacterium thermoautotrophicum $\Delta \mathrm{H}$. J. Bacteriol. 176: 6384-6391.

25. Reeve, J. N. 1992. Molecular biology of methanogens. Annu. Rev. Microbiol. 46:165-191.

26. Rivera, M., and J. A. Lake. 1996. The phylogeny of Methanopyrus kandleri. Int. J. Syst. Bacteriol. 46:348-351.

27. Rospert, S., D. Linder, J. Ellermann, and R. K. Thauer. 1990. Two genetically distinct methyl coenzyme $\mathbf{M}$ reductases in Methanobacterium thermoautotrophicum strains Marburg and $\Delta \mathrm{H}$. Eur. J. Biochem. 194:871-877.

28. Sambrook, J. E., E. F. Fritsch, and T. Maniatis. 1989. Molecular cloning: a laboratory manual, 2nd ed. Cold Spring Harbor Laboratory, Cold Spring Harbor, N.Y.

29. Shima, S., D. S. Weiss, and R. K. Thauer. 1995. Formylmethanofuran: tetrahydromethanopterin formyltransferase (Ftr) from the hyperthermophilic Methanopyrus kandleri. Cloning, sequencing and functional expression of the ftr gene and one-step purification of the enzyme overproduced in Escherichia coli. Eur. J. Biochem. 230:906-913.

30. Springer, E., M. S. Sachs, C. R. Woese, and D. R. Boone. 1995. Partial gene sequences for the A subunit on methyl-coenzyme $\mathrm{M}$ reductase $(\mathrm{mcrl})$ as a phylogenetic tool for the family Methanosarcinaceae. Int. J. Syst. Bacteriol. 45:554-559.

31. Stroup, D., and J. N. Reeve. 1993. Association of the $m c r D$ gene product with methyl coenzyme $\mathrm{M}$ reductase in Methanococcus vannielii. Biochim. Biophys. Acta 1203:175-183.

32. Thomas, L. K., D. B. Dix, and R. C. Thompson. 1988. Codon choice and gene expression: synonymous codons differ in their ability to direct aminoacylated transfer RNA binding to ribosomes in vitro. Proc. Natl. Acad. Sci. USA 85:4242-4246.

33. Weil, C. F., D. S. Cram, B. A. Sherf, and J. N. Reeve. 1988. Structure and comparative analysis of the genes encoding component $\mathrm{C}$ of methyl coenzyme $\mathrm{M}$ reductase in the extremely thermophilic archaebacterium Methanothermus fervidus. J. Bacteriol. 170:4718-4726.

34. Zirngibl, C., W. van Dongen, B. Schwörer, R. van Bünan, M. Richter, A. Klein, and R. K. Thauer. 1992. $\mathrm{H}_{2}$-forming methylenetetrahydromethanopterin dehydrogenase, a novel type of hydrogenase without iron-sulfur clusters in methanogenic archaea. Eur. J. Biochem. 208:511-520. 